\title{
ON CLASSICAL KRULL DIMENSION OF GROUP-GRADED RINGS
}

\begin{abstract}
A.V. KelareV
For any ring $R$ graded by a finite group, we give a bound on the classical Krull dimension of $R$ in terms of the dimension of the initial component $R_{e}$. It follows that if $R_{e}$ has finite classical Krull dimension, then the same is true of the whole ring $R$, too.
\end{abstract}

Let $G$ be a finite group with identity $e$. A ring $R$ is said to be $G$-graded if $R=\bigoplus_{g \in G} R_{g}$ is a direct sum of additive subgroups $R_{g}$ and $R_{g} R_{h} \subseteq R_{g h}$ for all $g, h \in G$.

There are many results relating properties of a group-graded ring $R=\bigoplus_{g \in G} R_{g}$ and its initial component $R_{e}$, where $e$ is the identity of the group (see $[5,7,8]$ and [9]). Ring-theoretic dimensions of group-graded rings have been considered by several authors (see, for example, Bell [1], Chin and Quinn [2], Cohen and Montgomery [3], Năstăsescu [6]).

Rings with Krull dimension form an important class and have many nice properties (see [5]). Suppose that the set $S=\operatorname{Spec}(R)$ of prime ideals of $R$ satisfies a.c.c. Define the sets $S_{\alpha}$ inductively. Let $S_{0}$ be the set of all maximal elements in $S$; and for each ordinal $\alpha$ denote by $S_{\alpha}$ the set of all $s \in S$ such that $t \in S, t>s$ implies $t \in S_{\beta}$ for some $\beta<\alpha$. Then there exists the least ordinal $\alpha$ such that $S_{\alpha}=S$. This ordinal is called the classical Krull dimension of $R$. If it is finite, then it is also equal to the right Krull dimension of $R$ defined on the lattice of right ideals of $R$ (see [5, Chapter 6]).

Denote by $c l-K-\operatorname{dim}(R)$ the classical Krull dimension of $R$. For any ordinal $\alpha$ and positive integer $n$, we introduce ordinals $\alpha_{n}$, setting $\alpha_{1}=\alpha+1, \alpha_{n+1}=$ $(\alpha+1)\left(\alpha_{n}+1\right)$. We shall use the results on prime ideals due to Cohen and Montgomery [3] and prove the following theorem.

Theorem 1. Let $G$ be a finite group with identity $e$ and $|G|=n$, and let $R=\bigoplus_{g \in G} R_{g}$ be a $G$-graded ring. If $R_{e}$ has classical Krull dimension $\alpha$, then $R$ has classical Krull dimension, too, and $c l-K-\operatorname{dim}(R)<\alpha_{n}$.

This theorem is related to an open question [4, Problem 5].

Received 4th April, 1996

Copyright Clearance Centre, Inc. Serial-fee code: 0004-9729/97 \$A2.00+0.00. 
It is interesting to note that the analogous assertion is not valid for Krull dimension defined on the lattice of right ideals. Indeed, if we take any group $G$ with identity $e$ and an element $g \neq e$ in $G$, and take a ring $R$ with zero multiplication which has no Krull dimension, then we can view $R$ as a group-graded ring with $R_{e}=0, R_{g}=R$, and $R_{h}=0$ for all $h \in G \backslash\{e, g\}$. [2, Example 2.4] shows that our theorem does not transfer to rings graded by infinite groups, even in the case of the infinite cyclic group.

We need the following lemma (see [3, Theorems 7.1 and 7.3], or [9, Theorem 17.9]).

LEMMA 2. [3]. Let $G$ be a finite group with identity $e$, and let $R$ be a $G$-graded ring.

(i) If $P$ is a prime ideal of $R$, then there exist $n \leqslant|G|$ primes $Q_{1}, Q_{2}, \ldots, Q_{n}$ of $R_{e}$ minimal over $P \cap R_{e}$, and we have $P \cap R_{e}=Q_{1} \cap Q_{2} \cap \ldots \cap Q_{n}$.

(ii) If $P \subseteq Q$ are prime ideals of $R$ and $P \neq Q$, then $P \cap R_{e} \neq Q \cap R_{e}$.

Proof of TheOREM 1: Suppose to the contrary that $R$ contains a strictly increasing chain of prime ideals $P_{1} \subset P_{2} \subset \ldots \subset P_{\alpha_{n}}$. Lemma 2(i) tells us that, for each $\gamma \leqslant \alpha_{n}$, there exists a finite set $S_{\gamma}$ of prime ideals of $R_{e}$ minimal over $R_{e} \cap P_{\gamma}$ and such that

$$
\bigcap_{P \in S_{\gamma}} P=R_{e} \cap P_{\gamma}
$$

and $\left|S_{\gamma}\right| \leqslant|G|=n$.

Put $S=\bigcup_{\gamma \leqslant \alpha_{n}} S_{\gamma}$. If a prime ideal contains an intersection of a finite number of ideals, then it contains at least one of them. Therefore, for any $\delta<\varepsilon \leqslant \alpha_{n}$ and $P \in S_{e}$, there exists $Q \in S_{\delta}$, such that $Q \subseteq P$.

For $\delta<\varepsilon \leqslant \alpha_{n}, Q \in S_{\delta}$, and $P \in S_{\varepsilon}$, we shall write

$$
Q \ll P
$$

if and only if, for all $\mu, \delta<\mu<\varepsilon$, we can fix $I_{\epsilon} \in S_{\mu}$ so that $I_{\mu} \subseteq I_{\nu}$ whenever $\delta \leqslant \mu \leqslant \nu \leqslant \varepsilon$, where $I_{\delta}=Q$ and $I_{\varepsilon}=P$.

We shall show by induction on $\gamma \leqslant \alpha_{n}$ that, for each $P \in S_{\gamma}$, there exists $Q \in S_{1}$ such that $Q \ll P$. The case of $\gamma=1$ is trivial. Suppose that this has been proved for all $\delta<\gamma$. Take any ideal $P \in S_{\gamma}$.

If $\gamma$ is not a limit ordinal, then there exists $\gamma-1$ and we can take $P^{\prime} \in S_{\gamma-1}$ such that $P^{\prime} \subseteq P$. By the induction assumption $Q \ll P^{\prime}$ for some $Q \in S_{1}$. It follows that $Q \ll P$.

Consider the case where $\gamma$ is a limit ordinal. Denote by $L$ the set of all $Q \in \bigcup_{\delta<\gamma} S_{\delta}$ such that $Q \subseteq P$. By induction on $\delta$ we shall define ideals $Q_{\delta} \in S_{\delta}$, for all $\delta<\gamma$. 
Given that $S_{1}$ is finite and every ideal $Q$, where $Q \in S_{\nu} \cap L \neq \emptyset, 1<\nu \leqslant \gamma$, contains at least one ideal of $S_{1} \cap L$, it follows that there exists $Q_{1} \in S_{1} \cap L$ such that for any $\mu<\gamma$ we can find $\mu<\nu<\gamma$ and $Q \in S_{\nu}$ satisfying $Q_{1} \ll Q$. By the definition of $\ll$, for any $\mu<\gamma$, we can find $Q \in S_{\mu}$ satisfying $Q_{1} \ll Q$. Put

$$
L_{1}=L \cap\left\{Q \in \bigcup_{1<\nu<\gamma} S_{\nu} \mid Q_{1} \ll Q\right\}
$$

We have ensured that $L_{1}$ intersects all $S_{\nu}$, for $1<\nu<\gamma$.

Suppose that for some $\delta<\gamma$ ideals $Q_{\varepsilon}$ have been defined for all $\varepsilon<\delta$, and suppose that these ideals form an ascending chain. In addition, assume that the sets

$$
L_{\varepsilon}=L \cap\left\{Q \in \bigcup_{e<\nu<\gamma} S_{\nu} \mid Q_{e} \ll Q\right\}
$$

intersect all $S_{\nu}$ for $\varepsilon<\nu<\gamma$. Obviously, $M \subseteq L$ and $M=\bigcap_{\varepsilon<\delta} L_{\epsilon} \cap S_{\delta}$ is not empty, because all sets $L_{1} \supseteq L_{2} \supseteq \ldots \supseteq L_{\delta} \supseteq \ldots$ are nonempty. As in the paragraph above, given that $M$ is finite, there exists $Q_{\delta} \in M$ such that for any $\delta<\mu<\gamma$ we can find $\mu<\nu<\gamma$ and $Q \in S_{\nu}$ satisfying $Q_{\delta} \subseteq Q$. Thus the ascending chain of ideals $Q_{\delta}$, $\delta<\gamma$, has been defined.

Since $Q_{\delta} \subseteq P$ for all $\delta<\gamma$, we see that $Q_{1} \ll P$, as required.

Next, we are going to reduce the set $S$. Take any $P^{(1)} \in S_{\alpha_{n}}$ and fix a chain of ideals $P_{\gamma}^{(1)} \in S_{\gamma}$ such that $P_{\mu}^{(1)} \subseteq P_{\nu}^{(1)}$ for all $\mu \leqslant \nu \leqslant \alpha_{n}$. Given that cl-K- $\operatorname{dim}\left(R_{e}\right)=$ $\alpha$ and $\alpha_{n}=(\alpha+1)\left(\alpha_{n-1}+1\right)$, there exists $0 \leqslant \delta<\alpha_{n}$ such that

$$
P_{\delta+1}^{(1)}=P_{\delta+2}^{(1)}=\cdots=P_{\delta+\alpha_{n-1}+1}^{(1)} \subseteq R_{e} .
$$

Put $S_{\mu}^{(1)}=S_{\mu} \backslash\left\{P_{\mu}^{(1)}\right\}$ for $\delta \leqslant \mu \leqslant \delta+\alpha_{n-1}+1$, and

$$
S^{(1)}=\bigcup_{\delta<\mu \leqslant \delta+\alpha_{n-1}} S_{\mu}^{(1)}
$$

For any $\delta<\mu<\nu \leqslant \delta+\alpha_{n-1}+1$, and any ideal $I \in S_{\nu}^{(1)}$ there exists $Q \in S_{\mu}$ such that $Q \subseteq I$. If $Q \notin S_{\mu}^{(1)}$, then $Q=P_{\mu}^{(1)}=P_{\nu}^{(1)}$; whence $I \supseteq P_{\nu}^{(1)}$, a contradiction. Therefore $Q \in S_{\mu}^{(1)}$.

Thus $S^{(1)}$ satisfies the same property we used for $S$, but now $\left|S_{\gamma}^{(1)}\right| \leqslant n-1$ for all $\gamma$.

Suppose that for some $\gamma$ such that $\delta<\gamma \leqslant \delta+\alpha_{n-1}$ the set $S_{\gamma}^{(1)}$ is empty. Then, for any $\gamma<\mu \leqslant \delta+\alpha_{n-1}+1$ and $Q \in S_{\mu}$, we have $P_{\mu}^{(1)}=P_{\gamma}^{(1)}=P_{\gamma} \subseteq Q$. Hence 
$Q=P_{\mu}^{(1)}$ and so $S_{\mu}^{(1)}=\emptyset$. Therefore $P_{\gamma}=P_{\gamma+1}$ by Lemma 2(ii). This contradiction shows that all sets $S_{\gamma}^{(1)}$ are nonempty for $\delta<\gamma \leqslant \delta+\alpha_{n-1}$.

Let us apply the same argument as above to $S^{(1)}$. Take an ideal $P^{(2)}$ in $S_{6+\alpha_{n-1}}^{(1)}$. Find $P_{\delta+1}^{(2)} \in S_{\delta+1}$ with $P_{\delta+1}^{(2)} \ll P^{(2)}$. Take a chain

$$
P_{\delta+1}^{(2)} \subseteq P_{\delta+2}^{(2)} \subseteq \ldots \subseteq P_{\delta+\alpha_{n-1}}^{(2)}=P^{(2)} \subseteq R_{e},
$$

where $P_{\gamma}^{(2)} \in S_{\gamma}^{(1)}$ for all $\delta<\gamma \leqslant \delta+\alpha_{n-1}$. Find a new ordinal $\delta_{2}$ such that $P_{\delta_{2}+1}^{(2)}=P_{\delta_{2}+2}^{(2)}=\cdots=P_{\delta_{2}+\alpha_{n-2}+1}^{(2)}$. Put $S_{\gamma}^{(2)}=S_{\gamma}^{(1)} \backslash\left\{P_{\gamma}^{(2)}\right\}$,

$$
S^{(2)}=\bigcup_{\delta_{2}<\gamma \leqslant \delta_{2}+\alpha_{n-2}} S_{\gamma}^{(2)} \text {. }
$$

Then the set $S^{(2)}$ satisfies the same property we used for $S$, but now $\left|S_{\gamma}^{(2)}\right| \leqslant n-2$ for all $\gamma$. As above, all sets $S_{\gamma}^{(2)}$ will be nonempty for $\delta_{2}<\gamma \leqslant \delta_{2}+\alpha_{n-2}$.

If we repeat this reduction $n-1$ times, we get a set

$$
S^{(n-1)}=\bigcup_{\delta_{n-1}<\gamma \leqslant \delta_{n-1}+\alpha_{1}} S_{\gamma}^{(n-1)}
$$

satisfying the same conditions and such that $\left|S_{\gamma}^{(n-1)}\right| \leqslant 1$ for all $\delta_{n-1}<\gamma \leqslant \delta_{n-1}+\alpha_{1}$. As earlier we can show that all sets $S_{\gamma}^{(n-1)}$ are nonempty for $\delta_{n-1}<\gamma \leqslant \delta_{n-1}+\alpha_{1}$. Thus $\left|S_{\gamma}^{(n-1)}\right|=1$ for all $\gamma$.

Given that $\alpha_{1}=\alpha+1$, we get $S_{\gamma}^{(n-1)}=S_{\gamma+1}^{n-1}$ for some $\delta_{2} \leqslant \gamma<\delta_{2}+\alpha_{1}$. It follows from Lemma 2(ii) that $P_{\gamma}=P_{\gamma+1}$. This contradiction completes the proof. REMARK. For a finite cl-K- $\operatorname{dim}\left(R_{e}\right)$ our proof simplifies since several steps become redundant.

\section{REFERENCES}

[1] A.D. Bell, 'Localization and ideal theory in Noetherian strongly group-graded rings', $J$. Algebra 105 (1987), 76-115.

[2] W. Chin and D. Quinn, 'Rings graded by polycyclic-by-fi nite groups', Proc. Amer. Math. Soc. 102 (1988), 235-241.

[3] M. Cohen and S. Montgomery, 'Group-graded rings, smas h products, and group actions', Trans. Amer. Math. Soc. 282 (1984), 237-258.

[4] A.V. Kelarev, 'Applications of epigroups to graded rin; theory', Semigroup Forum 50 (1995), 327-350. 
[5] J.C. McConnell and J.C. Robson, Noncommutative Noetherian rings (Wiley, New York, 1987).

[6] C. Năstăsescu, 'Strongly graded rings of finite groups', Comm. Algebra 11 (1983), 1033-1071.

[7] C. Năstăsescu and F. Van Oystaeyen, Graded ring theory, Math. Library 28 (North-Holland, Amsterdam, New York, 1982).

[8] C. Năstăsescu and F. Van Oystaeyen, Dimensions of ring theory (Reidel, Dordrecht, 1987).

[9] D.S. Passman, Infinite crossed products (Academic Press, New York, 1989).

Department of Mathematics

The University of Tasmania

GPO Box 252-37

Hobart Tas 7001

Australia

e-mail: kelarev@hilbert.maths.utas.edu.au 